\title{
ITERATED HYPER-EXTENSIONS AND AN IDEMPOTENT ULTRAFILTER PROOF OF RADO'S THEOREM
}

\author{
MAURO DI NASSO
}

(Communicated by Mirna Džamonja)

\begin{abstract}
By using nonstandard analysis, and in particular iterated hyperextensions, we give foundations to a peculiar way of manipulating ultrafilters on the natural numbers and their pseudo-sums. The resulting formalism is suitable for applications in Ramsey theory of numbers. To illustrate the use of our technique, we give a (rather) short proof of Milliken-Taylor's Theorem and a ultrafilter version of Rado's Theorem about partition regularity of diophantine equations.
\end{abstract}

\section{INTRODUCTION}

The algebraic structure on the space of ultrafilters $\beta \mathbb{N}$ as given by the pseudosum operation $\mathcal{U} \oplus \mathcal{V}$, and the related generalizations, have been deeply investigated during the last thirty years, revealing a powerful tool for applications in Ramsey theory and combinatorial number theory (see the monograph [15]). The aim of this paper is to introduce a peculiar formalism grounded on the use of the hyper-natural numbers of nonstandard analysis that allows us to manipulate ultrafilters on $\mathbb{N}$ and their pseudo-sums in a simplified manner. In particular, we shall be interested in linear combinations $a_{0} \mathcal{U} \oplus \ldots \oplus a_{k} \mathcal{U}$ of a given idempotent ultrafilter $\mathcal{U}$. To illustrate the use of our technique, we shall give a nonstandard proof of Ramsey's Theorem and a (rather) short proof of Milliken-Taylor's Theorem, a strengthening of the celebrated Hindman's Theorem. Moreover, we shall also prove the following ultrafilter version of Rado's Theorem that seems to be new.

Theorem. Let $c_{1} X_{1}+\ldots+c_{k} X_{k}=0$ be a diophantine equation with $c_{1}+\ldots+c_{k}=0$ and $k>2$. Then there exists $a_{0}, \ldots, a_{k-2} \in \mathbb{N}$ such that for every idempotent ultrafilter $\mathcal{U}$, the corresponding linear combination

$$
\mathcal{V}=a_{0} \mathcal{U} \oplus \ldots \oplus a_{k-2} \mathcal{U}
$$

witnesses the injective partition regularity of the given equation, i.e. for every $A \in \mathcal{V}$ there exist distinct elements $x_{1}, \ldots, x_{k} \in A$ with $c_{1} x_{1}+\ldots+c_{k} x_{k}=0$.

At the end of the paper, some hints are given for further possible applications and developments of the introduced nonstandard technique.

We assume the reader to be familiar with the notion of ultrafilter and with the basics of nonstandard analysis. In particular, we shall call a star map or nonstandard embedding a function $A \mapsto{ }^{*} A$ that associates to each mathematical object $A$

Received by the editors April 12, 2013 and, in revised form, July 30, 2013.

2010 Mathematics Subject Classification. Primary 03H05; Secondary 03E05, 05D10, 11 D04.

Key words and phrases. Nonstandard analysis, ultrafilters, Ramsey theory, diophantine equations. 
under consideration its hyper-extension ${ }^{*} A$, and that satisfies the transfer principle. Excellent references for the foundations of nonstandard analysis are [6], $\S 4.4$, where the classical superstructure approach is presented, and the textbook [12], grounded on the ultrapower construction. The peculiarity of our nonstandard approach is that we shall use iterated hyper-extensions (see the discussion in Section 2).

\section{1. $u$-EQUIVALENT POLYNOMIALS}

Before starting to work in a nonstandard setting, in this section we present a general result about linear combinations of a given idempotent ultrafilter (see below for the definition), whose proof will be given in Section 4. As a consequence, we prove an ultrafilter version of Rado's Theorem.

Throughout the paper, $\mathbb{N}$ will denote the set of positive integers, and $\mathbb{N}_{0}=\mathbb{N} \cup\{0\}$ the set of nonnegative integers.

Recall the pseudo-sum operation between ultrafilters on $\mathbb{N}_{0}$ :

$$
A \in \mathcal{U} \oplus \mathcal{V} \Longleftrightarrow\left\{n \in \mathbb{N}_{0} \mid A-n \in \mathcal{V}\right\} \in \mathcal{U},
$$

where $A-n=\left\{m \in \mathbb{N}_{0} \mid m+n \in A\right\}$ is the left-ward shift of $A$ by $n$. It can be readily verified that $\mathcal{U} \oplus \mathcal{V}$ is actually an ultrafilter, and that the pseudo-sum operation is associative. If one identifies every principal ultrafilter $\mathfrak{U}_{n}=\left\{A \subseteq \mathbb{N}_{0} \mid\right.$ $n \in A\}$ with its generator $n \in \mathbb{N}_{0}$, then it is readily seen that the pseudo-sum extends the usual addition, i.e. $\mathfrak{U}_{n} \oplus \mathfrak{U}_{m}=\mathfrak{U}_{n+m} ;$ moreover, $\mathcal{U} \oplus \mathfrak{U}_{0}=\mathfrak{U}_{0} \oplus \mathcal{U}=\mathcal{U}$ for all $\mathcal{U}$. (In fact, it can be proved that the center of $\left(\beta \mathbb{N}_{0}, \oplus\right)$ is the family $\left\{\mathfrak{U}_{n} \mid n \in \mathbb{N}_{0}\right\}$ of the principal ultrafilters. A nonstandard proof of this fact can be found in [11.)

Given an ultrafilter $\mathcal{U}$ on $\mathbb{N}$ and a natural number $h$, the product $h \mathcal{U}$ is the ultrafilter defined by

$$
A \in h \mathcal{U} \Longleftrightarrow A / h=\{n \mid n h \in A\} \in \mathcal{U} .
$$

Notice that $0 \mathcal{U}=\mathfrak{U}_{0}$ and $1 \mathcal{U}=\mathcal{U}$ for every $\mathcal{U}$.

Particularly relevant for applications are the idempotent ultrafilters, namely the nonprincipal ultrafilters $\mathcal{U}$ such that $\mathcal{U} \oplus \mathcal{U}=\mathcal{U}$. We remark that their existence is a nontrivial result whose proof requires repeated applications of Zorn's lemma. (To be precise, also the principal ultrafilter $\mathfrak{U}_{0}$ has the property $\mathfrak{U}_{0}=\mathfrak{U}_{0} \oplus \mathfrak{U}_{0}$, but it is not usually considered as an "idempotent" in the literature.)

Let us now introduce an equivalence relation on the strings (i.e. finite sequences) of integers.

Definition 1.1. The u-equivalence $\approx$ u between strings of integers is the smallest equivalence relation such that:

- The empty string $\varepsilon \approx\langle\widetilde{u}\langle 0\rangle$.

- $\langle a\rangle \approx\langle\widetilde{u}\langle a, a\rangle$ for all $a \in \mathbb{Z}$.

- $\approx$ is coherent with concatenations 1 i.e.

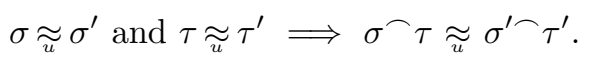

Two polynomials $P(X)=\sum_{i=0}^{n} a_{i} X^{i}$ and $Q(X)=\sum_{j=0}^{m} b_{j} X^{j}$ in $\mathbb{Z}[X]$ are $u$ equivalent when the corresponding strings of coefficients are $u$-equivalent:

$$
\left.\left\langle a_{0}, \ldots, a_{n}\right\rangle \approx b_{0}, \ldots, b_{m}\right\rangle
$$

\footnotetext{
${ }^{1}$ Recall that if $\sigma=\left\langle a_{0}, \ldots, a_{k}\right\rangle$ and $\tau=\left\langle b_{0}, \ldots, b_{h}\right\rangle$, then their concatenation is defined as $\sigma \frown \tau=\left\langle a_{0}, \ldots, a_{k}, b_{0}, \ldots, b_{h}\right\rangle$.
} 
So, $\widetilde{u}$-equivalence between strings is preserved by inserting or removing zeros, by repeating finitely many times a term or, conversely, by shortening a block of consecutive equal terms; e.g. $\langle 3,0,0,-4,1,1\rangle \widetilde{\widetilde{u}}\langle 0,3,-4,-4,1\rangle$ and $\langle 2,2,0,0,7,7,3\rangle \widetilde{\widetilde{u}}$ $\langle 2,7,3\rangle$, and hence:

- $X^{5}+X^{4}-4 X^{3}+3 \approx X^{4}-4 X^{3}-4 X^{2}+3 X$,

- $3 X^{6}+7 X^{5}+7 X^{4}+2 X+2 \approx 3 X^{2}+7 X+2$, etc.

As an application of nonstandard analysis, in Section 4 the following will be proved:

Theorem 4.4. Let $a_{0}, \ldots, a_{n} \in \mathbb{N}_{0}$, and assume that there exist [distinct] polynomials $P_{i}(X)$ such that

$$
P_{1}(X) \underset{\widetilde{u}}{\approx} \ldots P_{k}(X) \underset{\widetilde{u}}{\approx} \sum_{i=0}^{n} a_{i} X^{i} \text { and } c_{1} P_{1}(X)+\ldots+c_{k} P_{k}(X)=0 .
$$

Then for every idempotent ultrafilter $\mathcal{U}$ and for every $A \in a_{0} \mathcal{U} \oplus \ldots \oplus a_{n} \mathcal{U}$, there exist [distinct] $x_{i} \in A$ such that $c_{1} x_{1}+\ldots+c_{k} x_{k}=0$.

We derive here a straight consequence of the above theorem which is an ultrafilter version of Rado's Theorem.

Theorem 1.2. Let $c_{1} X_{1}+\ldots+c_{k} X_{k}=0$ be a diophantine equation with $c_{1}+$ $\ldots+c_{k}=0$ and $k>2$. Then there exists $a_{0}, \ldots, a_{k-2} \in \mathbb{N}$ such that for every idempotent ultrafilter $\mathcal{U}$, the corresponding linear combination

$$
\mathcal{V}=a_{0} \mathcal{U} \oplus \ldots \oplus a_{k-2} \mathcal{U}
$$

witnesses the injective partition regularity of the given equation, i.e. for every $A \in \mathcal{V}$ there exist distinct $x_{i} \in A$ such that $c_{1} x_{1}+\ldots+c_{k} x_{k}=0$.

Proof. For arbitrary $a_{0}, \ldots, a_{k-2}$, consider the following polynomials:

$$
\begin{aligned}
& P_{1}(X)=a_{0}+a_{1} X+a_{2} X^{2}+\ldots+a_{k-3} X^{k-3}+a_{k-2} X^{k-2}+a_{k-2} X^{k-1}
\end{aligned}
$$

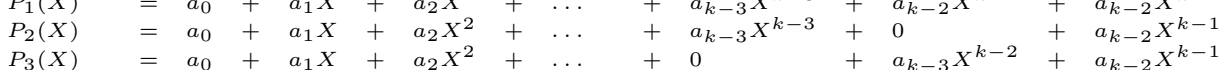

$$
\begin{aligned}
& \begin{array}{l}
\vdots \\
P_{k-2}(X)=a_{0}+a_{1} X+a_{0}+a_{2} X^{3}+
\end{array} \\
& \begin{array}{l}
P_{k-1}(X)=a_{0}+a_{1}+a_{0}+a_{1}+a_{2} X^{3}+\ldots \\
+a_{1} X^{2}+\ldots
\end{array} \\
& P_{k}(X)=a_{0}+a_{0} X+a_{1} X^{2}+a_{2} X^{3}+\ldots+a_{k-3} X^{k-2}+a_{k-2} X^{k-1}
\end{aligned}
$$

Notice that $P_{1}(X) \approx \ldots \approx \widetilde{u} P_{k}(X) \approx \sum_{i=0}^{k-2} a_{i} X^{i}$. In order to apply Theorem 4.4. we need to find suitable coefficients $a_{0}, \ldots, a_{k-2}$ in such a way that the linear combination $c_{1} P_{1}(X)+\ldots+c_{k} P_{k}(X)=0$. It is readily verified that this happens if and only if the following conditions are fulfilled:

$$
\left\{\begin{array}{l}
\left(c_{1}+\ldots+c_{k}\right) \cdot a_{0}=0 \\
\left(c_{1}+\ldots+c_{k-2}\right) \cdot a_{1}+c_{k} \cdot a_{0}=0 \\
\left(c_{1}+\ldots+c_{k-3}\right) \cdot a_{2}+\left(c_{k-1}+c_{k}\right) \cdot a_{1}=0 \\
\quad \vdots \\
c_{1} \cdot a_{k-2}+\left(c_{3}+\ldots+c_{k}\right) \cdot a_{k-3}=0 \\
\left(c_{1}+\ldots+c_{k}\right) \cdot a_{k-2}=0
\end{array}\right.
$$

The first and the last equations are trivially satisfied because of the hypothesis $c_{1}+\ldots+c_{k}=0$. Now assume without loss of generality that the coefficients $c_{1} \geq$ $\ldots \geq c_{k}$ are arranged in nonincreasing order. It can be verified in a straightforward 
manner that the remaining $k-2$ equations are satisfied by (infinitely many) suitable $a_{0}, \ldots, a_{k-2} \in \mathbb{N}$, e.g.:

$$
\left\{\begin{array}{l}
a_{0}=c_{1} \cdot\left(c_{1}+c_{2}\right) \cdot \ldots \cdot\left(c_{1}+\ldots+c_{k-2}\right) \\
a_{i}=b_{i} \cdot b_{i}^{\prime} \text { for } 0<i<k-2 \text { where } \\
\quad b_{i}=c_{1} \cdot\left(c_{1}+c_{2}\right) \cdot \ldots \cdot\left(c_{1}+\ldots+c_{k-2-i}\right) \text { and } \\
\quad b_{i}^{\prime}=(-1)^{i} \cdot c_{k} \cdot\left(c_{k}+c_{k-1}\right) \cdot \ldots \cdot\left(c_{k}+\ldots+c_{k+1-i}\right) \\
a_{k-2}=(-1)^{k-2} \cdot c_{k} \cdot\left(c_{k}+c_{k-1}\right) \cdot \ldots \cdot\left(c_{k}+\ldots+c_{3}\right)
\end{array}\right.
$$

Remark that by the hypothesis $c_{1}+\ldots+c_{k}=0$ together with the assumption $c_{1} \geq \ldots \geq c_{k}$, it follows that all $a_{i}>0$. Finally, remark also that since all coefficients $a_{0}, \ldots, a_{k-2} \neq 0$, the polynomials $P_{i}(X)$ are mutually distinct.

For instance, consider the diophantine equation

$$
3 X_{1}+X_{2}+X_{3}-X_{4}-4 X_{5}=0
$$

where coefficients are arranged in nonincreasing order and their sum equals zero. By using $(*)$ in the above proof, we obtain that $a_{0}=60, a_{1}=48, a_{2}=60, a_{3}=80$. So, for every idempotent ultrafilter $\mathcal{U}$ and for every set

$$
A \in 60 \mathcal{U} \oplus 48 \mathcal{U} \oplus 60 \mathcal{U} \oplus 80 \mathcal{U}
$$

we know that there exist distinct $x_{i} \in A$ such that $3 x_{1}+x_{2}+x_{3}-x_{4}-4 x_{5}=0$.

\section{ITERATED HYPER-EXTENSIONS}

For our purposes, we shall need to work in models of nonstandard analysis where hyper-extensions can be iterated, so that one can consider, e.g., the set of hyper-hyper-natural numbers ${ }^{* *} \mathbb{N}$, the hyper-hyper-hyper-natural numbers ${ }^{* * *} \mathbb{N}$, the hyper-extension ${ }^{*} \nu$ of a hyper-natural number $\nu$, and so forth. Moreover, we shall use the $\mathfrak{c}^{+}$-enlargement property, namely the property that intersections $\bigcap_{F \in \mathcal{F}}{ }^{*} F$ are nonempty for all families $|\mathcal{F}| \leq \mathfrak{c}$ of cardinality at most the continuum, and which satisfy the finite intersection property (i.e. $A_{1} \cap \ldots \cap A_{n} \neq \emptyset$ for every choice of finitely many $A_{i} \in \mathcal{F}$ ).

In full generality, all the above requirements are fulfilled by taking a $\mathfrak{c}^{+}$-enlarging nonstandard embedding

$$
*: \mathbf{V} \longrightarrow \mathbf{V}
$$

which is defined on the universal class $\mathbf{V}$ of all sets. It is now a well-known fact in nonstandard set theory that such "universal" nonstandard embeddings can be constructed within conservative extensions of ZFC where the regularity axiom is replaced by a suitable anti-foundation axiom; see e.g. [1]2 (For a comprehensive treatment of nonstandard set theories, we refer the interested reader to the monograph [16.)

A suitable axiomatic framework is the nonstandard set theory $\mathrm{ZFC}[\Omega]$ of $[10$, which includes all axioms of Zermelo-Fraenkel theory ZFC with the only exception of the regularity axiom, and where for every " $\in$-definable" cardinal $\kappa$, one has a nonstandard embedding $J_{\kappa}: \mathbf{V} \rightarrow \mathbf{V}$ of the universe into itself that satisfies the

\footnotetext{
${ }^{2}$ In summary, one takes transitive Mostowski collapses of ultrapowers $\mathbf{V}^{I} / \mathcal{U}$ of the universe. For any given cardinal $\kappa$, the $\kappa$-enlargement property is obtained by picking a $\kappa$-regular ultrafilter $\mathcal{U}$.
} 
$\kappa$-enlargement property 3 The resulting theory is conservative over ZFC. (See also the related axiomatics *ZFC [7,8] and Alpha-Theory [9].)

Another suitable setting where iterated hyper-extensions can be considered was introduced by $\mathrm{V}$. Benci in [2]: it consists of a special version of the superstructure approach $*: V(X) \rightarrow V(X)$ where the standard universe and the nonstandard universe coincide. The limitation here is that superstructures $V(X)$ only satisfy a fragment of ZFC (e.g., replacement fails and there are no infinite ordinals in $V(X)$ ).

We stress that working with iterated hyper-extensions requires caution. To begin with, recall that in nonstandard analysis one has that ${ }^{*} n=n$ for all natural numbers $n \in \mathbb{N}$; however, the same property cannot be extended to the hyper-natural numbers $\xi \in{ }^{*} \mathbb{N}$. Indeed, by transfer one can easily show that ${ }^{*} \xi>\xi$ for all infinite $\xi \in{ }^{*} \mathbb{N}$; more generally, the following facts hold:

- $* \mathbb{N} \varsubsetneqq * * \mathbb{N}$.

- If $\xi \in{ }^{*} \mathbb{N} \backslash \mathbb{N}$, then ${ }^{*} \xi \in{ }^{* *} \mathbb{N} \backslash * \mathbb{N}$.

- ${ }^{*} \mathbb{N}$ is an initial segment of ${ }^{*} \mathbb{N}$, i.e. $\xi<\nu$ for every $\xi \in{ }^{*} \mathbb{N}$ and for every $\nu \in{ }^{* *} \mathbb{N} \backslash * \mathbb{N}$.

Let $f: \mathbb{N}_{0} \rightarrow \mathbb{N}_{0}$. By transferring the fact that ${ }^{*} f$ and $f$ agree on $\mathbb{N}_{0}$, one gets that

- $\left({ }^{* *} f\right)(\xi)={ }^{*} f(\xi)$ for all $\xi \in{ }^{*} \mathbb{N}_{0}$.

Remark that ${ }^{*}\left[{ }^{*} f(\xi)\right]=\left({ }^{* *} f\right)\left({ }^{*} \xi\right)$, but in general $\left({ }^{* *} f\right)\left({ }^{*} \xi\right) \neq\left({ }^{* *} f\right)(\xi)$.

Now denote by " $k *$ " the $k$-times iterated star map, i.e.

$$
\left\{\begin{array}{l}
{ }^{0 *} A=A, \\
{ }^{(k+1) *} A=*\left({ }^{k *} A\right) .
\end{array}\right.
$$

As a first application of iterated hyper-extensions, we now present a nonstandard proof of Ramsey's Theorem.

Lemma 2.1. Let $A \subseteq \mathbb{N}^{k}$. For any infinite $\xi \in{ }^{*} \mathbb{N}$ such that $\left(\xi,{ }^{*} \xi, \ldots,{ }^{(k-1) *} \xi\right) \in$ ${ }^{k *} A$ there exists an infinite set of natural numbers

$$
H=\left\{h_{1}<h_{2}<\ldots<h_{n}<h_{n+1}<\ldots\right\}
$$

such that $\left(h_{n_{1}}, h_{n_{2}}, \ldots, h_{n_{k}}\right) \in A$ for all $n_{1}<n_{2}<\ldots<n_{k}$.

Proof. To simplify notation, let us only consider here the particular case $k=3$. The general result is proved exactly in the same fashion, only by using heavier notation. So, let us assume that $\left(\xi,{ }^{*} \xi,{ }^{* *} \xi\right) \in{ }^{* * *} A$. For $n, n^{\prime} \in \mathbb{N}$, let:

- $X=\left\{n \in \mathbb{N} \mid\left(n, \xi,{ }^{*} \xi\right) \in{ }^{* *} A\right\}$;

- $X_{n}=\left\{n^{\prime} \in \mathbb{N} \mid\left(n, n^{\prime}, \xi\right) \in *^{*} A\right\}$;

- $X_{n n^{\prime}}=\left\{n^{\prime \prime} \in \mathbb{N} \mid\left(n, n^{\prime}, n^{\prime \prime}\right) \in A\right\}$.

The corresponding hyper-extensions are described as follows:

- ${ }^{*} X=\left\{\eta \in{ }^{*} \mathbb{N} \mid\left(\eta,{ }^{*} \xi,{ }^{* *} \xi\right) \in{ }^{* * *} A\right\}$;

- ${ }^{*} X_{n}=\left\{\eta \in{ }^{*} \mathbb{N} \mid\left(n, \eta,{ }^{*} \xi\right) \in{ }^{* *} A\right\}$;

- ${ }^{*} X_{n, n^{\prime}}=\left\{\eta \in{ }^{*} \mathbb{N} \mid\left(n, n^{\prime}, \eta\right) \in{ }^{*} A\right\}$.

Notice that $n \in X \Leftrightarrow \xi \in{ }^{*} X_{n}$, and $n^{\prime} \in X_{n} \Leftrightarrow \xi \in{ }^{*} X_{n n^{\prime}}$. By the hypothesis, we have that $\xi \in{ }^{*} X$, so $X$ is an infinite set and we can pick an element $h_{1} \in X$. Now, $\xi \in{ }^{*} X \cap{ }^{*} X_{h_{1}}$ implies that $X \cap X_{h_{1}}$ is infinite, and so we can pick an element

\footnotetext{
${ }^{3}$ Indeed, the separation and replacement schemas hold for all $\in$-*-formulas, and $J_{\kappa}$ is postulated to satisfy $\kappa$-saturation, a stronger property than $\kappa$-enlargement.
} 
$h_{2}>h_{1}$ in that intersection. But then $\xi \in{ }^{*} X \cap{ }^{*} X_{h_{1}} \cap{ }^{*} X_{h_{2}} \cap{ }^{*} X_{h_{1} h_{2}}$, and so we can pick an element $h_{3}>h_{2}$ in the intersection $X \cap X_{h_{1}} \cap X_{h_{2}} \cap X_{h_{1} h_{2}}$. In particular, $\left(h_{1}, h_{2}, h_{3}\right) \in A$. An increasing sequence $\left\langle h_{n} \mid n \in \mathbb{N}\right\rangle$ that satisfies the desired property is obtained by iterating this procedure, where at each step $n$ one has

$$
\xi \in{ }^{*} X \cap \bigcap_{1 \leq i \leq n}{ }^{*} X_{h_{i}} \cap \bigcap_{1 \leq i<j \leq n}{ }^{*} X_{h_{i} h_{j}},
$$

and $h_{n+1}>h_{n}$ is picked in the infinite intersection

$$
h_{n+1} \in X \cap \bigcap_{1 \leq i \leq n} X_{h_{i}} \cap \bigcap_{1 \leq i<j \leq n} X_{h_{i} h_{j}} .
$$

As a straight corollary, one obtains:

Theorem 2.2 (Ramsey). Let $[\mathbb{N}]^{k}=C_{1} \sqcup \ldots \sqcup C_{r}$ be a finite partition of the $k$-sets of natural numbers 4 Then there exists an infinite $H \subseteq \mathbb{N}$ such that all its $k$-sets are monochromatic, i.e. $[H]^{k} \subseteq C_{i}$ for some $i$.

Proof. Identify the family of $k$-sets $[\mathbb{N}]^{k}$ with the upper-diagonal in the Cartesian product $\mathbb{N}^{k}$ :

$$
\left\{\left(n_{1}, n_{2}, \ldots, n_{k}\right) \in \mathbb{N}^{k} \mid n_{1}<n_{2}<\ldots<n_{k}\right\} .
$$

By applying transfer to the $k$-iterated star map, one gets that ${ }^{k *}\left(\left[{ }^{*} \mathbb{N}\right]^{k}\right)=\left[{ }^{k *} \mathbb{N}\right]^{k}$, and one has the following finite coloring:

$$
\left[{ }^{k *} \mathbb{N}\right]^{k}={ }^{k *} C_{1} \sqcup \ldots \sqcup{ }^{k *} C_{r} .
$$

Now fix any infinite $\xi \in{ }^{*} \mathbb{N}$, and let $i$ be such that the ordered $k$-tuple $\left(\xi,{ }^{*} \xi, \ldots,{ }^{(k-1) *} \xi\right) \in{ }^{k *} C_{i}$ (notice that $\xi<{ }^{*} \xi<\ldots<{ }^{(k-1) *} \xi$ ). By Lemma 2.1, we get the existence of an infinite $H \subseteq \mathbb{N}$ such that $[H]^{k} \subseteq\left[C_{i}\right]^{k}$.

\section{Hyper-natural nUmbers as REPRESENTATIVES OF ULTRAFILTERS}

Recall that there is a canonical way of associating an ultrafilter to every element $\alpha \in{ }^{*} \mathbb{N}_{0}$ (see e.g. [5, 11, 19]). Namely, one takes the family of those sets of natural numbers whose hyper-extensions contain $\alpha$ :

$$
\mathfrak{U}_{\alpha}=\left\{A \subseteq \mathbb{N}_{0} \mid \alpha \in{ }^{*} A\right\} .
$$

It is readily verified from the properties of hyper-extensions that $\mathfrak{U}_{\alpha}$ is indeed an ultrafilter, called the ultrafilter generated by $\alpha$. Notice that $\mathfrak{U}_{\alpha}$ is principal if and only if $\alpha \in \mathbb{N}_{0}$ is finite. Notice also that if $\mathcal{U}=\mathfrak{U}_{\alpha}$ is generated by $\alpha$, then $h \mathcal{U}$ is generated by $h \alpha$.

Definition 3.1. We say that two elements $\alpha, \beta \in{ }^{*} \mathbb{N}_{0}$ are $u$-equivalent, and write $\alpha \widetilde{u} \beta$, if they generate the same ultrafilter: $\mathfrak{U}_{\alpha}=\mathfrak{U}_{\beta}$.

So, $\alpha \widetilde{u} \beta$ when $\alpha \in{ }^{*} A \Leftrightarrow \beta \in{ }^{*} A$ for all $A \subseteq \mathbb{N}_{0}$. Since every ultrafilter $\mathcal{U}$ on $\mathbb{N}_{0}$ is a family of $\mathfrak{c}$-many sets with the finite intersection property, by the hypothesis of $\mathfrak{c}^{+}$-enlargement there exists an element $\alpha \in \bigcap_{A \in \mathcal{U}}{ }^{*} A$. This means that every ultrafilter $\mathcal{U}=\mathfrak{U}_{\alpha}$ is generated by some number $\alpha \in *^{*} \mathbb{N}_{0}$.

\footnotetext{
${ }^{4} \mathrm{~A} k$-set is a set with exactly $k$-many elements.
} 
The following properties are readily verified:

Proposition 3.2. Let $\alpha \widetilde{u} \alpha^{\prime}$ be two u-equivalent hyper-natural numbers, and let $n \in \mathbb{N}$ be finite. Then:

(1) $\alpha \pm n \widetilde{u} \alpha^{\prime} \pm n$

(2) $n \cdot \alpha \widetilde{u} n \cdot \alpha^{\prime}$,

(3) $\alpha / n \widetilde{u} \alpha^{\prime} / n$, provided $\alpha$ is divisible by $n$.

Remark that in general sums in ${ }^{*} \mathbb{N}_{0}$ are not coherent with $u$-equivalence, i.e. it can well be the case that $\alpha \widetilde{u} \alpha^{\prime}$ and $\beta \widetilde{u} \beta^{\prime}$, but $\alpha+\beta \Varangle_{u} \alpha^{\prime}+\beta^{\prime}$.

We now extend the notion of generated ultrafilter and also consider elements $\nu \in{ }^{k *} \mathbb{N}$ in iterated hyper-extensions of $\mathbb{N}$, by putting

$$
\mathfrak{U}_{\nu}=\left\{A \subseteq \mathbb{N}_{0} \mid \nu \in{ }^{k *} A\right\} .
$$

The $u$-equivalence relation is extended accordingly to all pairs of numbers in the following union:

$$
{ }^{\star} \mathbb{N}_{0}=\bigcup_{k \in \mathbb{N}}{ }^{k *} \mathbb{N}_{0}
$$

In general, for every $A \subseteq \mathbb{N}$, the set ${ }^{\star} A=\bigcup_{k \in \mathbb{N}}{ }^{k *} A$ can be seen as the direct limit of the finitely iterated hyper-extensions of $A$; and similarly for functions. In consequence, the map $\star$ itself is a nonstandard embedding, i.e. it satisfies the transfer principle. Since $*$ is assumed to be $\mathfrak{c}^{+}$-enlarging, it can be easily verified that the same property also holds for $\star$.

Remark that the above definitions are coherent. In fact, by starting from the equivalence $n \in A \Leftrightarrow n \in{ }^{*} A$ which holds for all $n \in \mathbb{N}_{0}$, one can easily show that $\nu \in{ }^{k *} A \Leftrightarrow \nu \in{ }^{h *} A$ for all $\nu \in{ }^{k *} \mathbb{N}_{0}$ and $h>k$. In consequence, for all $\nu \in{ }^{\star} \mathbb{N}_{0}$, one has $\nu \widetilde{u}^{*} \nu$, and hence ${ }^{k *} \nu \widetilde{u}^{h *} \nu$ for all $k, h$. (A detailed study of $\widetilde{u}^{\prime}$-equivalence in ${ }^{\star} \mathbb{N}_{0}$ can be found in [17].)

We shall use the following characterization of pseudo-sums of ultrafilters.

Proposition 3.3. Let $\alpha, \beta \in{ }^{*} \mathbb{N}_{0}$ and $A \subseteq \mathbb{N}_{0}$. Then $A \in \mathfrak{U}_{\alpha} \oplus \mathfrak{U}_{\beta}$ if and only if the sum $\alpha+{ }^{*} \beta \in{ }^{* *} A$.

Proof. Consider the set $\widehat{A}=\left\{n \in \mathbb{N}_{0} \mid A-n \in \mathfrak{U}_{\beta}\right\}$, and notice that its hyperextension ${ }^{*} \widehat{A}={ }^{*}\left\{n \in \mathbb{N}_{0} \mid n+\beta \in{ }^{*} A\right\}=\left\{\gamma \in{ }^{*} \mathbb{N}_{0} \mid \gamma+{ }^{*} \beta \in{ }^{* *} A\right\}$. Then the following equivalences yield the thesis:

$$
A \in \mathfrak{U}_{\alpha} \oplus \mathfrak{U}_{\beta} \Longleftrightarrow \widehat{A} \in \mathfrak{U}_{\alpha} \Longleftrightarrow \alpha \in^{*} \widehat{A} \Longleftrightarrow \alpha+{ }^{*} \beta \in^{* *} A .
$$

We already mentioned that when $\alpha \widetilde{u} \alpha^{\prime}$ and $\beta \widetilde{u} \beta^{\prime}$, one cannot conclude that $\alpha+\beta \chi_{u} \alpha^{\prime}+\beta^{\prime}$. However, under the same assumptions, one has that $\alpha+{ }^{*} \beta \widetilde{u} \alpha+{ }^{*} \beta^{\prime}$ in ${ }^{* *} \mathbb{N}$, as they generate the same ultrafilter $\mathfrak{U}_{\alpha} \oplus \mathfrak{U}_{\beta}=\mathfrak{U}_{\alpha^{\prime}} \oplus \mathfrak{U}_{\beta^{\prime}}$.

The characterization of pseudo-sums as given above can be extended to linear combinations of ultrafilters in a straightforward manner.

Corollary 3.4. For every $\xi_{0}, \ldots, \xi_{k} \in{ }^{*} \mathbb{N}_{0}$, and for every $a_{0}, \ldots, a_{k} \in \mathbb{N}_{0}$, the linear combination $a_{0} \mathcal{U} \oplus \ldots \oplus a_{k} \mathcal{U}$ is the ultrafilter generated by the element $a_{0} \xi+$ $\ldots+a_{k}{ }^{k *} \xi \in{ }^{(k+1) *} \mathbb{N}_{0}$.

The class of idempotent ultrafilters was first isolated to provide a simplified proof of Hindman's Theorem, a cornerstone of combinatorics of numbers. 
Theorem (Hindman). For every finite coloring $\mathbb{N}=C_{1} \sqcup \ldots \sqcup C_{r}$ there exists an infinite set $X=\left\{x_{1}<x_{2}<\ldots<x_{n}<\ldots\right\}$ such that all its finite sums are monochromatic, i.e. there exists $i$ such that:

$$
F S(X)=\left\{\sum_{i \in F} x_{i} \mid F \subset \mathbb{N} \text { nonempty finite }\right\} \subseteq C_{i} .
$$

Starting from the ultrafilter proof of the above theorem, a whole body of new combinatorial results have been then obtained by exploiting the algebraic properties of the space $\left(\beta \mathbb{N}_{0}, \oplus\right)$ and of its generalizations (see the monograph [15]).

By Proposition 3.3, it directly follows that

Proposition 3.5. Let $\xi \in{ }^{*} \mathbb{N}$. The ultrafilter $\mathfrak{U}_{\xi}$ is idempotent if and only if $\xi \widetilde{u} \xi+{ }^{*} \xi$.

Next, we show a general result connecting linear combinations of a given idempotent ultrafilter, and $u$-equivalence of the corresponding strings of coefficients.

Theorem 3.6. Let $a_{0}, a_{1}, \ldots, a_{k}, b_{0}, b_{1}, \ldots, b_{h} \in \mathbb{N}_{0}$. Then the following are equivalent:

(1) $\left.\left\langle a_{0}, a_{1}, \ldots, a_{k}\right\rangle \approx b_{0}, b_{1}, \ldots, b_{h}\right\rangle$.

(2) For every idempotent ultrafilter $\mathcal{U}$ :

$$
a_{0} \mathcal{U} \oplus a_{1} \mathcal{U} \oplus \ldots \oplus a_{k} \mathcal{U}=b_{0} \mathcal{U} \oplus b_{1} \mathcal{U} \oplus \ldots \oplus b_{h} \mathcal{U}
$$

(3) For every $\xi \in{ }^{*} \mathbb{N}_{0}$ such that the generated ultrafilter $\mathfrak{U}_{\xi}$ is idempotent:

$$
a_{0} \xi+a_{1}{ }^{*} \xi+\ldots+a_{k}{ }^{k *} \xi \widetilde{u} b_{0} \xi+b_{1}{ }^{*} \xi+\ldots+b_{h}{ }^{h *} \xi .
$$

Proof. (1) $\Rightarrow(2)$. For every string $\sigma=\left\langle d_{0}, d_{1}, \ldots, d_{n}\right\rangle$ of numbers $d_{i} \in \mathbb{N}_{0}$, and for every idempotent ultrafilter $\mathcal{U}$, denote by

$$
\oplus \mathcal{U}(\sigma)=d_{0} \mathcal{U} \oplus d_{1} \mathcal{U} \oplus \ldots \oplus d_{n} \mathcal{U}
$$

We have to show that $\sigma \widetilde{\widetilde{u}} \tau \Rightarrow \oplus_{\mathcal{U}}(\sigma)=\oplus_{\mathcal{U}}(\tau)$. By agreeing that $\oplus_{\mathcal{U}}(\varepsilon)=\mathfrak{U}_{0}$, one trivially has $\oplus_{\mathcal{U}}(\varepsilon)=\oplus_{\mathcal{U}}(\langle 0\rangle)$. Moreover, $\oplus(\langle a\rangle)=\oplus_{\mathcal{U}}(\langle a, a\rangle)$ because $a \mathcal{U} \oplus a \mathcal{U}=$ $a(\mathcal{U} \oplus \mathcal{U})=a \mathcal{U}$. Now let $\sigma \widetilde{\widetilde{u}} \sigma^{\prime}$ and $\tau \approx \tau^{\prime}$, where we assume by inductive hypothesis that $\oplus_{\mathcal{U}}(\sigma)=\oplus_{\mathcal{U}}\left(\sigma^{\prime}\right)$ and $\oplus_{\mathcal{U}}(\tau)=\oplus_{\mathcal{U}}\left(\tau^{\prime}\right)$. Then, by associativity of the pseudosum, it follows that

$$
\oplus_{\mathcal{U}}\left(\sigma^{\frown} \tau\right)=\left[\oplus_{\mathcal{U}}(\sigma)\right] \oplus\left[\oplus_{\mathcal{U}}(\tau)\right]=\left[\oplus_{\mathcal{U}}\left(\sigma^{\prime}\right)\right] \oplus\left[\oplus_{\mathcal{U}}\left(\tau^{\prime}\right)\right]=\oplus_{\mathcal{U}}\left(\sigma^{\prime} \tau^{\prime}\right) .
$$

$(2) \Rightarrow(1)$. Assume that $\left\langle a_{0}, a_{1}, \ldots, a_{k}\right\rangle \underset{\widetilde{u}}{\approx}\left\langle b_{0}, b_{1}, \ldots, b_{h}\right\rangle$. By the previous implication, we can assume without loss of generality that $a_{i} \neq a_{i+1}$ for $i<k$, and that $b_{j} \neq b_{j+1}$ for $j<h$. Then we apply the following known result:

- $\left(\left[18\right.\right.$, Theorem 2.19) Let $a_{0}, \ldots, a_{k}, b_{0}, \ldots, b_{h} \in \mathbb{N}$ so that $a_{i} \neq a_{i+1}$ and $b_{j} \neq b_{j+1}$ for any $i<k$ and $j<h$. If $a_{0} \mathcal{U} \oplus \ldots \oplus a_{k} \mathcal{U}=b_{0} \mathcal{U} \oplus \ldots \oplus b_{h} \mathcal{U}$ for some idempotent $\mathcal{U}$, then $k=h$ and $a_{i}=b_{i}$ for all $i$.

$(2) \Leftrightarrow(3)$. By the $\mathfrak{c}^{+}$-enlargement property, every ultrafilter $\mathcal{U}$ is generated by some element $\xi \in{ }^{*} \mathbb{N}$, i.e. $\mathcal{U}=\mathfrak{U}_{\xi}$. So, the thesis is a particular case of Corollary 3.4 .

We shall use the above characterization to justify a neat formalism which is suitable to handle idempotent ultrafilters and their linear combinations. As a first relevant example, let us give a nonstandard ultrafilter proof of Milliken-Taylor's Theorem, a strengthening of Hindman's Theorem. 
Lemma 3.7. Let $\mathcal{U}$ be an idempotent ultrafilter, and let $a_{0}, \ldots, a_{k} \in \mathbb{N}$. For every $A \in a_{0} \mathcal{U} \oplus \ldots \oplus a_{k} \mathcal{U}$ there exists an infinite set of natural numbers

$$
X=\left\{x_{1}<x_{2}<\ldots<x_{n}<\ldots\right\}
$$

with the property that for every increasing sequence $I_{0}<\ldots<I_{k}$ of nonempty finite sets of natural numbers (i.e. $\max I_{i}<\min I_{i+1}$ ), the sum

$$
\sum_{i \in I_{0}} a_{0} x_{i}+\ldots+\sum_{i \in I_{k}} a_{k} x_{i} \in A
$$

Proof. By the $\mathfrak{c}^{+}$-enlargement property, we can pick $\xi \in{ }^{*} \mathbb{N}$ with $\mathcal{U}=\mathfrak{U}_{\xi}$. By Corollary 3.4 .

$$
A \in a_{0} \mathcal{U} \oplus \ldots \oplus a_{k} \mathcal{U} \Longleftrightarrow a_{0} \xi+a_{1}{ }^{*} \xi+\ldots+a_{k}{ }^{k *} \xi \in{ }^{(k+1) *} A,
$$

and so we have that

$$
\begin{aligned}
\xi & \in\left\{\eta \in{ }^{*} \mathbb{N} \mid a_{0} \eta+a_{1}{ }^{*} \xi+\ldots+a_{k}{ }^{k *} \xi \in{ }^{(k+1) *} A\right\} \\
& =*\left\{x \in \mathbb{N} \mid a_{0} x+a_{1} \xi+\ldots+a_{k}{ }^{(k-1) *} \xi \in{ }^{k *} A\right\} .
\end{aligned}
$$

Since $\left\langle a_{0}, a_{1}, \ldots, a_{k}\right\rangle \widetilde{\widetilde{u}}\left\langle a_{0}, a_{0}, a_{1}, \ldots, a_{k}\right\rangle$, we also have that $a_{0} \xi+a_{0}{ }^{*} \xi+a_{1}{ }^{* *} \xi+$ $\ldots+a_{k}{ }^{(k+1) *} \xi \in{ }^{(k+2) *} A$, and hence:

$$
\begin{aligned}
\xi & \in\left\{\eta \in{ }^{*} \mathbb{N} \mid a_{0} \eta+a_{0}{ }^{*} \xi+a_{1}{ }^{* *} \xi+\ldots+a_{k}{ }^{(k+1) *} \xi \in{ }^{(k+2) *} A\right\} \\
& =*\left\{x \in \mathbb{N} \mid a_{0} x+a_{0} \xi+a_{1}{ }^{*} \xi+\ldots+a_{k}{ }^{k *} \xi \in{ }^{(k+1) *} A\right\} .
\end{aligned}
$$

By transfer, there exists an element $x_{1}$ such that

- $a_{0} x_{1}+a_{1} \xi+\ldots+a_{k}(k-1) * \xi \in{ }^{k *} A$, and

- $a_{0} x_{1}+a_{0} \xi+a_{1}{ }^{*} \xi+\ldots+a_{k}^{k *} \xi \in{ }^{(k+1) *} A$.

We now proceed by induction on $n$ and define elements $x_{1}<\ldots<x_{n}$ in such a way that for every increasing sequence of nonempty finite sets $J_{0}<\ldots<J_{h}$ where $h \leq k$ and $\max J_{h} \leq n$, the following properties are fulfilled:

(1) $\sum_{s=0}^{h}\left(\sum_{i \in J_{s}} a_{s} x_{i}\right)+a_{h} \xi+a_{h+1}{ }^{*} \xi+\ldots+a_{k}{ }^{(k-h) *} \xi \in{ }^{(k-h+1) *} A$.

(2) $\sum_{s=0}^{h}\left(\sum_{i \in J_{s}} a_{s} x_{i}\right)+a_{h+1} \xi+a_{h+2}{ }^{*} \xi+\ldots+a_{k}{ }^{(k-h-1) *} \xi \in{ }^{(k-h) *} A$.

Remark that $x_{1}$ actually satisfies the inductive basis $n=1$, because in this case one necessarily has $h=0$ and $J_{0}=\{1\}$. As for the inductive step, notice that

- $\left\langle a_{h}, a_{h+1}, \ldots, a_{k}\right\rangle \underset{\widetilde{u}}{\widetilde{2}}\left\langle a_{h}, a_{h}, a_{h+1}, \ldots, a_{k}\right\rangle$, and

- $\left\langle a_{h+1}, a_{h+2}, \ldots, a_{k}\right\rangle \underset{\widetilde{u}}{\widetilde{u}}\left\langle a_{h+1}, a_{h+1}, a_{h+2}, \ldots, a_{k}\right\rangle$.

So, in consequence of the inductive hypotheses (1) and (2) respectively, one has that

(3) $\sum_{s=0}^{h}\left(\sum_{i \in J_{s}} a_{s} x_{i}\right)+a_{h} \xi+a_{h}{ }^{*} \xi+a_{h+1}{ }^{* *} \xi+\ldots+a_{k}{ }^{(k-h+1) *} \xi \in^{(k-h+2) *} A$.

(4) $\sum_{s=0}^{h}\left(\sum_{i \in J_{s}} a_{s} x_{i}\right)+a_{h+1} \xi+a_{h+1}{ }^{*} \xi+a_{h+2}{ }^{* *} \xi+\ldots+a_{k}{ }^{(k-h) *} \xi \in{ }^{(k-h+1) *} A$. 
Now, properties $(1),(2),(3),(4)$ say that for every increasing sequence of nonempty finite sets $J_{0}<\ldots<J_{h}$ where $h \leq k$ and $\max J_{h} \leq n$, the hyperinteger $\xi \in{ }^{*} \Gamma\left(J_{0}<\ldots<J_{h}\right)$ where:

$$
\begin{aligned}
& \Gamma\left(J_{0}<\ldots<J_{h}\right) \\
&=\left\{m \in \mathbb{N} \mid \sum_{s=0}^{h}\left(\sum_{i \in J_{s}} a_{s} x_{i}\right)+a_{h} m+a_{h+1} \xi+\ldots+a_{k}{ }^{(k-h-1) *} \xi \in{ }^{(k-h) *} A\right. \\
& \& \sum_{s=0}^{h}\left(\sum_{i \in J_{s}} a_{s} x_{i}\right)+a_{h+1} m+a_{h+2} \xi+\ldots+a_{k}{ }^{(k-h-2) *} \xi \in{ }^{(k-h-1) *} A \\
& \& \sum_{s=0}^{h}\left(\sum_{i \in J_{s}} a_{s} x_{i}\right)+a_{h} m+a_{h} \xi+a_{h+1}{ }^{*} \xi+\ldots+a_{k}{ }^{(k-h) *} \xi \in{ }^{(k-h+1) *} A \\
&\left.\& \sum_{s=0}^{h}\left(\sum_{i \in J_{s}} a_{s} x_{i}\right)+a_{h+1} m+a_{h+1} \xi+a_{h+2}{ }^{*} \xi+\ldots+a_{k}{ }^{(k-h-1) *} \xi \in(k-h) * A\right\} .
\end{aligned}
$$

Then $\xi \in{ }^{*} \Gamma$, where $\Gamma$ is the following finite intersection:

$$
\Gamma=\bigcap_{\substack{J_{0}<\ldots<J_{h} \\ h \leq k, \max J_{h} \leq n}} \Gamma\left(J_{0}<\ldots<J_{h}\right) .
$$

The set $\Gamma$ is infinite because its hyper-extension contains an infinite hyper-natural number, namely $\xi$; in particular, we can pick an element $x_{n+1}>x_{n}$ in $\Gamma$. It now only takes a straightforward verification to check that $x_{1}<\ldots<x_{n}<x_{n+1}$ satisfy the desired properties, namely (1) and (2), for every sequence of nonempty finite sets $J_{0}<\ldots<J_{l}$ where $l \leq k$ and $\max J_{l} \leq n+1$.

As a straight corollary, we obtain

Theorem 3.8 (Milliken-Taylor). Let a finite coloring $\mathbb{N}=C_{1} \sqcup \ldots \sqcup C_{r}$ be given. For every choice of $a_{0}, \ldots, a_{k} \in \mathbb{N}$ there exists an infinite set

$$
X=\left\{x_{1}<x_{2}<\ldots<x_{n}<x_{n+1}<\ldots\right\}
$$

such that the following sums are monochromatic for every increasing sequence $I_{0}<$ $\ldots<I_{k}$ of nonempty finite sets:

$$
\sum_{i \in I_{0}} a_{0} x_{i}+\ldots+\sum_{i \in I_{k}} a_{k} x_{i} .
$$

Proof. Pick any idempotent ultrafilter $\mathcal{U}$, and consider the linear combination $\mathcal{W}=$ $a_{0} \mathcal{U} \oplus \ldots \oplus a_{k} \mathcal{U}$. Then take $i$ such that $C_{i} \in \mathcal{W}$, and apply the previous lemma.

\section{Partition Regularity and Rado's Theorem}

We now aim at showing how the introduced nonstandard approach can be used in partition regularity of linear equations. Let us start with an example. Recall the following known fact.

Theorem 4.1 (4], Th. 2.10). Let $\mathcal{U}$ be an idempotent ultrafilter. Then every set $A \in 2 \mathcal{U} \oplus \mathcal{U}$ contains a 3 -term arithmetic progression. 
A nonstandard proof of the above theorem is obtained by the following simple observation. If the ultrafilter $\mathcal{U}=\mathfrak{U}_{\xi}$ is idempotent, then the following three elements of the hyper-hyper-hyper-natural numbers ${ }^{* * *} \mathbb{N}$ are arranged in arithmetic progression, and they all generate the same ultrafilter $\mathcal{W}=2 \mathcal{U} \oplus \mathcal{U}$ :

- $\nu=2 \xi+0+{ }^{* *} \xi$,

- $\mu=2 \xi+{ }^{*} \xi+{ }^{* *} \xi$,

- $\lambda=2 \xi+2^{*} \xi+{ }^{* *} \xi$.

The property that $\nu \widetilde{u} \mu_{u} \lambda \widetilde{u}_{u} 2 \xi+{ }^{*} \xi$ directly follows from Theorem [3.6, since $\langle 2,0,1\rangle \approx\langle 2,1,1\rangle \approx\langle 2,2,1\rangle \approx\langle 2,1\rangle$. Moreover, by Proposition 3.3. the generated ultrafilter is the following:

$$
\mathcal{W}=\mathcal{U}_{2 \xi+{ }^{*} \xi}=\mathfrak{U}_{2 \xi} \oplus \mathfrak{U}_{\xi}=2 \mathcal{U} \oplus \mathcal{U} .
$$

If $A \in \mathcal{W}$, then $\nu, \mu, \lambda \in{ }^{* * *} A$, and the existence of a 3 -term arithmetic progression in $A$ is proved by applying backward transfer to the following property, which holds in ${ }^{* * *} \mathbb{N}$ :

$$
\exists x, y, z \in{ }^{* * *} A \text { s.t. } y-x=z-y>0 .
$$

We now elaborate on this example to prove a general fact which connects partition regularity of equations with $u$-equivalence in the direct limit

$$
{ }^{\star} \mathbb{N}=\bigcup_{k \in \mathbb{N}} k * \mathbb{N} .
$$

Recall the following

Definition 4.2. An equation $F\left(X_{1}, \ldots, X_{n}\right)=0$ is [injectively] partition regular on $\mathbb{N}_{0}$ if for every finite coloring of $\mathbb{N}_{0}=C_{1} \sqcup \ldots \sqcup C_{r}$ there exist [distinct] monochromatic elements $x_{1}, \ldots, x_{n}$ which are a solution, i.e. $F\left(x_{1}, \ldots, x_{n}\right)=0$ and $x_{1}, \ldots, x_{n} \in C_{i}$ for a suitable color $C_{i}$.

It is a well-known fact that partition regularity is intimately connected with ultrafilters. In particular, recall the following:

- $F\left(X_{1}, \ldots, X_{n}\right)=0$ is [injectively] partition regular on $\mathbb{N}_{0}$ if and only if there exists an ultrafilter $\mathcal{V}$ on $\mathbb{N}_{0}$ such that in every $A \in \mathcal{V}$ one finds [distinct] elements $x_{1}, \ldots, x_{n} \in A$ with $F\left(x_{1}, \ldots, x_{n}\right)=05$

When the above property is satisfied, we say that the ultrafilter $\mathcal{V}$ is a witness of the [injective] partition regularity of $F\left(X_{1}, \ldots, X_{n}\right)=0$.

A useful nonstandard characterization holds.

Theorem 4.3. Let the nonstandard embedding $*$ satisfy the $\mathfrak{c}^{+}$-enlargement property. Then an ultrafilter $\mathcal{V}$ on $\mathbb{N}_{0}$ witnesses the [injective] partition regularity of the equation $F\left(X_{1}, \ldots, X_{n}\right)=0$ if and only if there exist [distinct] hyper-natural numbers $\xi_{1}, \ldots, \xi_{n} \in{ }^{*} \mathbb{N}_{0}$ such that $\mathcal{V}=\mathfrak{U}_{\xi_{1}}=\ldots=\mathfrak{U}_{\xi_{n}}$ and ${ }^{*} F\left(\xi_{1}, \ldots, \xi_{n}\right)=0$.

Proof. Assume first that the ultrafilter $\mathcal{V}$ is a witness. For $A \in \mathcal{V}$, let

$$
\Gamma(A)=\left\{\left(x_{1}, \ldots, x_{n}\right) \in A^{n} \mid\left[x_{i} \neq x_{j} \text { for } i \neq j\right] \& F\left(x_{1}, \ldots, x_{n}\right)=0\right\} .
$$

Since $\Gamma(A) \cap \Gamma(B)=\Gamma(A \cap B)$, by the hypothesis it follows that the family $\{\Gamma(A) \mid$ $A \in \mathcal{V}\}$ satisfies the finite intersection property and hence, by $\mathfrak{c}^{+}$-enlargement, we can pick $\left(\xi_{1}, \ldots, \xi_{n}\right) \in \bigcap_{A \in \mathcal{V}}{ }^{*} \Gamma(A)$. Then it is readily checked from the definitions

\footnotetext{
${ }^{5} \mathrm{~A}$ proof of this equivalence can be found e.g. in 15 , §3.1.
} 
that the [distinct] components $\xi_{1}, \ldots, \xi_{n}$ are such that $\mathfrak{U}_{\xi_{1}}=\ldots=\mathfrak{U}_{\xi_{n}}=\mathcal{V}$ and ${ }^{*} F\left(\xi_{1}, \ldots, \xi_{n}\right)=0$.

Conversely, let $A \in \mathcal{V}=\mathfrak{U}_{\xi_{1}}=\ldots=\mathfrak{U}_{\xi_{n}}$. By applying backward transfer to the property: "There exist [distinct] $\xi_{1}, \ldots, \xi_{n} \in{ }^{*} A$ such that ${ }^{*} F\left(\xi_{1}, \ldots, \xi_{n}\right)=0$ ", one obtains the existence of [distinct] $x_{1}, \ldots, x_{n} \in A$ such that $F\left(x_{1}, \ldots, x_{n}\right)=0$, as desired.

We can finally prove the result that was used in Section 1 to prove an ultrafilter version of Rado's Theorem, namely Theorem 1.2 ,

Theorem 4.4. Let $a_{0}, \ldots, a_{n} \in \mathbb{N}_{0}$, and assume that there exist [distinct] polynomials $P_{i}(X)$ such that

$$
P_{1}(X) \underset{\widetilde{u}}{\approx} \approx \underset{\widetilde{u}}{\approx} P_{k}(X) \underset{\widetilde{u}}{\approx} \sum_{i=0}^{n} a_{i} X^{i} \text { and } c_{1} P_{1}(X)+\ldots+c_{k} P_{k}(X)=0 .
$$

Then for every idempotent ultrafilter $\mathcal{U}$ and for every $A \in a_{0} \mathcal{U} \oplus \ldots \oplus a_{n} \mathcal{U}$, there exist [distinct] $x_{i} \in A$ such that $c_{1} x_{1}+\ldots+c_{k} x_{k}=0$.

Proof. For $i=1, \ldots, k$, let the polynomial $P_{i}(X)=\sum_{j=0}^{n_{i}} b_{i j} X^{j}$ correspond to the string of coefficients $\left\langle b_{i 0}, b_{i 1}, \ldots, b_{i n_{i}}\right\rangle$. Given the idempotent ultrafilter $\mathcal{U}$, pick a hyper-natural number $\xi \in{ }^{*} \mathbb{N}$ such that $\mathfrak{U}_{\xi}=\mathcal{U}$ (this is always possible by $\mathfrak{c}^{+}$enlargement), and consider the numbers

$$
\zeta_{i}=b_{i 0} \xi+b_{i 1}{ }^{*} \xi+\ldots+b_{i n_{i}}{ }^{n^{*}} \xi \in{ }^{\left(n_{i}+1\right) *} \mathbb{N} \subset{ }^{\star} \mathbb{N} .
$$

Since $\xi^{*} \mathbb{N}$ is infinite, for every $d \in \mathbb{N}_{0}$ one has $d<\xi, d \xi<{ }^{*} \xi, d^{*} \xi<{ }^{* *} \xi$, and so forth. In consequence, by the hypothesis $c_{1} P_{1}(X)+\ldots+c_{k} P_{k}(X)=0$, it directly follows that $c_{1} \zeta_{1}+\ldots+c_{k} \zeta_{k}=0$. Moreover, by the hypotheses $P_{i}(X) \approx \sum_{i=0}^{n} a_{i} X^{i}$, Theorem 3.6 guarantees that

$$
\mathfrak{U}_{\zeta_{1}}=\ldots=\mathfrak{U}_{\zeta_{n}}=a_{0} \mathcal{U} \oplus \ldots \oplus a_{n} \mathcal{U}
$$

The thesis is finally reached applying the previous Theorem 4.3. (Recall that the nonstandard embedding $\star$ is $\mathfrak{c}^{+}$-enlarging, because the starting nonstandard embedding $*$ was.)

\section{FinAL REMARKS}

A similar characterization of partition regularity as the one given in Theorem 4.3 can also be proved for (possibly infinite) systems of equations. It seems worth investigating the use of such nonstandard characterizations especially for the study of homogeneous nonlinear equations (along the lines of [17]) and of infinite systems, which are research areas where very little is known (see [3, 13, 14]). In particular, also the notion of image partition regularity would deserve attention. Another possible direction for further research is to consider possible extensions of Theorem 1.2 which are closer to the most general form of Rado's Theorem for systems.

\section{REFERENCES}

[1] David Ballard and Karel Hrbáček, Standard foundations for nonstandard analysis, J. Symbolic Logic 57 (1992), no. 2, 741-748, DOI 10.2307/2275304. MR1169206 (93d:03071)

[2] Vieri Benci, A construction of a nonstandard universe, Advances in dynamical systems and quantum physics (Capri, 1993), World Sci. Publ., River Edge, NJ, 1995, pp. 11-21. MR.1414687 (97i:03040) 
[3] Ben Barber, Neil Hindman, and Imre Leader, Partition regularity in the rationals, J. Combin. Theory Ser. A 120 (2013), no. 7, 1590-1599, DOI 10.1016/j.jcta.2013.05.011. MR3092686

[4] Vitaly Bergelson and Neil Hindman, Nonmetrizable topological dynamics and Ramsey theory, Trans. Amer. Math. Soc. 320 (1990), no. 1, 293-320, DOI 10.2307/2001762. MR.982232 (90k:03046)

[5] Greg Cherlin and Joram Hirschfeld, Ultrafilters and ultraproducts in non-standard analysis, Contributions to non-standard analysis (Sympos., Oberwolfach, 1970), North-Holland, Amsterdam, 1972, pp. 261-279. Studies in Logic and Found. Math., Vol. 69. MR0485344 (58 \#5191)

[6] C. C. Chang and H. J. Keisler, Model Theory, 3rd ed., Studies in Logic and the Foundations of Mathematics, vol. 73, North-Holland Publishing Co., Amsterdam, 1990. MR.1059055 (91c:03026)

[7] Mauro Di Nasso, *ZFC: an axiomatic * approach to nonstandard methods (English, with English and French summaries), C. R. Acad. Sci. Paris Sér. I Math. 324 (1997), no. 9, 963967, DOI 10.1016/S0764-4442(97)87868-8. MR1451233(97m:03097)

[8] Mauro Di Nasso, An axiomatic presentation of the nonstandard methods in mathematics, J. Symbolic Logic 67 (2002), no. 1, 315-325, DOI 10.2178/jsl/1190150046. MR 1889553 (2002k:03089)

[9] Vieri Benci and Mauro Di Nasso, Alpha-theory: an elementary axiomatics for nonstandard analysis, Expo. Math. 21 (2003), no. 4, 355-386, DOI 10.1016/S0723-0869(03)80038-5. MR2022004 (2004i:03108)

[10] Mauro Di Nasso, ZFC[ $\Omega]:$ A nonstandard set theory where all sets have nonstandard extensions (abstract), Bull. Symb. Logic, vol. 7, 2001, p. 138.

[11] Mauro Di Nasso, Hyper-natural numbers as ultrafilters, unpublished manuscript.

[12] Robert Goldblatt, Lectures on the Hyperreals, Graduate Texts in Mathematics, vol. 188, Springer-Verlag, New York, 1998. An introduction to nonstandard analysis. MR.1643950 (2000a:03113)

[13] Neil Hindman, Imre Leader, and Dona Strauss, Open problems in partition regularity, Combin. Probab. Comput. 12 (2003), no. 5-6, 571-583, DOI 10.1017/S0963548303005716. Special issue on Ramsey theory. MR2037071 (2005e:05147)

[14] Neil Hindman, Imre Leader, and Dona Strauss, Infinite partition regular matrices: solutions in central sets, Trans. Amer. Math. Soc. 355 (2003), no. 3, 1213-1235, DOI 10.1090/S00029947-02-03191-4. MR.1938754(2003h:05187)

[15] N. Hindman and D. Strauss, Algebra in the Stone-Čech Compactification, Theory and Applications (2nd edition), W. de Gruyter, 2011.

[16] Vladimir Kanovei and Michael Reeken, Nonstandard Analysis, Axiomatically, Springer Monographs in Mathematics, Springer-Verlag, Berlin, 2004. MR2093998 (2006e:03001)

[17] L. Luperi Baglini, Hyperintegers and Nonstandard Techniques in Combinatorics of Numbers, Ph.D. dissertation, 2012, University of Siena, arXiv:1212.2049.

[18] Amir Maleki, Solving equations in $\beta \mathbb{N}$, Semigroup Forum 61 (2000), no. 3, 373-384, DOI 10.1007/PL00006036. MR1832314(2002c:54021)

[19] Siu-Ah Ng and Hermann Render, The Puritz order and its relationship to the RudinKeisler order, Reuniting the Antipodes-constructive and nonstandard views of the continuum (Venice, 1999), Synthese Lib., vol. 306, Kluwer Acad. Publ., Dordrecht, 2001, pp. 157-166. MR.1895391(2003d:54045)

Dipartimento di Matematica, Università di Pisa, Largo Bruno Pontecorvo 5, 56127 PisA, ITALY

E-mail address: dinasso@dm.unipi.it 